Article

\title{
An Efficient Chemical Synthesis of Scutellarein: An in Vivo Metabolite of Scutellarin
}

\author{
Ze-Xi Dong ${ }^{1}$, Nian-Guang $\mathrm{Li}^{1}{ }^{1,}$, Peng-Xuan Zhang ${ }^{1}$, Ting Gu ${ }^{1}$, Wen-Yu Wu ${ }^{1}$ and \\ Zhi-Hao Shi ${ }^{2, *}$ \\ 1 Jiangsu Collaborative Innovation Center of Chinese Medicinal Resources Industrialization, \\ Jiangsu Key Laboratory for High Technology Research of TCM Formulae, National and Local Collaborative \\ Engineering Center of Chinese Medicinal Resources Industrialization and Formulae Innovative Medicine, \\ Nanjing University of Chinese Medicine, Nanjing 210023, Jiangsu, China; dongzexi1215@163.com (Z.-X.D.); \\ zpx20130901@126.com (P.-X.Z.); guting1992@163.com (T.G.); 15150513147@163.com (W.-Y.W.) \\ 2 Department of Organic Chemistry, China Pharmaceutical University, Nanjing 211198, Jiangsu, China \\ * Correspondence: linianguang@njutcm.edu.cn (N.-G.L.); sszh163@163.com (Z.-H.S.); \\ Tel./Fax: +86-25-8581-1916 (N.-G.L.); +86-25-8618-5172 (Z.-H.S.)
}

Academic Editors: Derek J. McPhee and Wim Dehaen

Received: 24 January 2016 ; Accepted: 22 February 2016 ; Published: 25 February 2016

\begin{abstract}
Scutellarein (2), which is an important in vivo metabolite of scutellarin (1), was synthesized from 3,4,5-trimethoxyphenol (3) in high yield in four steps. This strategy relies on acetylation, aldolization, cyclization and hydrolysis reactions, respectively.
\end{abstract}

Keywords: scutellarin; scutellarein; metabolite; efficient; synthesis

\section{Introduction}

As a frequently-occurring disease, ischemic cerebrovascular is a serious threat to human health and it has been one of the leading causes of death and disability around the world [1]. Traditional Chinese medicines are rich sources for drug lead compound discovery as they have been used clinically for thousands years. Scutellarin (1, Figure 1), a main active ingredient extracted from Erigeron breviscapus (Vant.) Hand-Mazz., which mainly grows in Yunnan Province of China, has been wildly used to treat acute cerebral infarction and paralysis induced by cerebrovascular diseases such as hypertension, cerebral thrombosis and cerebral hemorrhage in China since 1984 [2]. Interestingly, scutellarin (1) is mainly hydrolyzed into scutellarein (2, Figure 1) in the intestine [3], and scutellarein (2) was much more easily absorbed than scutellarin (1) after oral administration of both of them in equal doses [4]. In previous studies, our research group has found that scutellarein (2) had better protective effects than scutellarin (1) against neuronal injury in a rat cerebral ischemia model $[5,6]$.
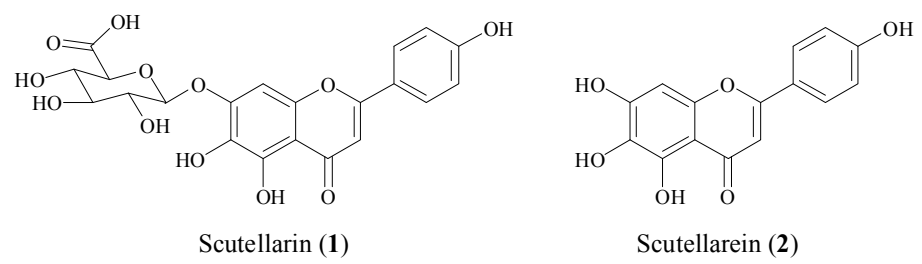

Figure 1. Chemical structures of scutellarin (1) and scutellarein (2).

Unfortunately, scutellarein (2) is not readily available commercially, so the chemical synthesis of this metabolite has become important in recent years. We have previously synthesized scutellarein 
(2) from scutellarin (1) by hydrolysis with $6 \mathrm{~N} \mathrm{HCl}$ in $90 \%$ ethanol under reflux, however, the yield was very low (only 17\%) [7]. Cui et al. [8] completed one synthetic route to scutellarein (2) from 2-hydroxy-4,5,6-trimethoxyacetophenone (4) and 4-methoxybenzoyl chloride in three steps, unfortunately, there was a by-product in this acetylation and the yield was low. Yen et al. [9] synthesized scutellarein (2) in 47\% yield in five steps starting from 3,4,5-trimethoxyphenol (3) and acetic anhydride, then the obtained 3,4,5-trimethoxyphenol acetate was transformed into 1-(6-hydroxy-2,3,4-trimethoxyphenyl)ethanone (4) after Fries rearrangement.

In this paper, we report an efficient chemical synthesis of scutellarein (2) from 3,4,5-trimethoxyphenol (3) and acetic acid in only four steps and with high total yield (58\%).

\section{Results and Discussion}

As shown in Scheme 1, the starting material 3,4,5-trimethoxyphenol (3, $200 \mathrm{mg}, 1.09 \mathrm{mmol})$ was first reacted with acetic acid $(1 \mathrm{~mL})$ in boron trifluoride diethyl etherate $(5 \mathrm{~mL})$ under a $\mathrm{N}_{2}$ atmosphere at $85{ }^{\circ} \mathrm{C}$, to afford a high yield $(92 \%)$ of the desired Friedel Crafts acylation reaction product 4 . Next, compound 5 was synthesized by a base-catalyzed Claisen-Schmidt condensation reaction of 4 and 4-methoxybenzaldehyde. Fortunately, the cyclization of 5 produced the desired compound 6 in $82 \%$ yield, using iodine as the catalyst in dimethyl sulfoxide solution at $100{ }^{\circ} \mathrm{C}$ for $2 \mathrm{~h}$. Finally, the demethylation of 6 with $40 \% \mathrm{HBr}$ in the refluxing $\mathrm{CH}_{3} \mathrm{COOH}$ led to scutellarein (2) in $90 \%$ yield, for a total overall yield of $58 \%$.

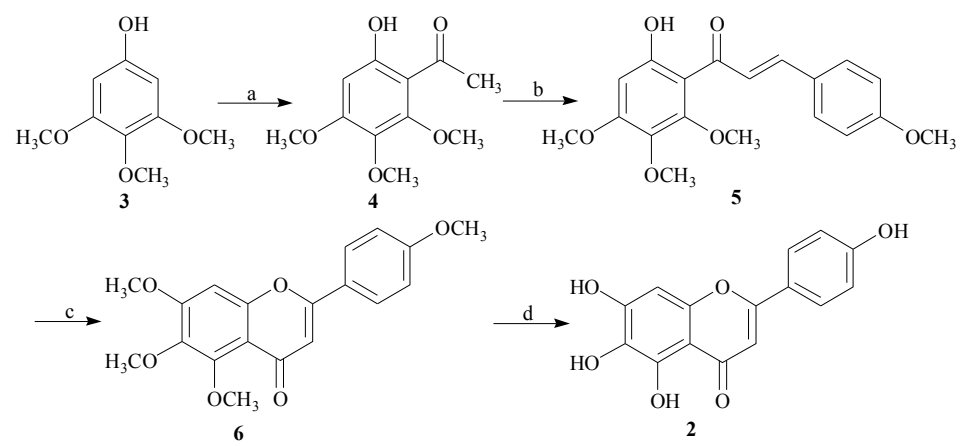

Reagents and Conditions: (a) $\mathrm{CH}_{3} \mathrm{COOH}, \mathrm{BF}_{3} \cdot \mathrm{Et}_{2} \mathrm{O}, \mathrm{N}_{2}$, reflux, $85^{\circ} \mathrm{C}, 2.5 \mathrm{~h}, 92 \%$; (b) 4-methoxybenzaldehyde (1.5 equiv), $t$-BuOK (2.5 equiv), EtOH, reflux, $85^{\circ} \mathrm{C}, 4 \mathrm{~h}, 86 \%$; (c) DMSO, $\mathrm{I}_{2}$, reflux, $100{ }^{\circ} \mathrm{C}, 2 \mathrm{~h}, 82 \%$; (d) $40 \%$ hydrobromic acid, $\mathrm{CH}_{3} \mathrm{COOH}, \mathrm{N}_{2}$, reflux, heat, $24 \mathrm{~h}, 90 \%$.

Scheme 1. The efficient chemical synthesis of scutellarein (2).

\section{Experimental Section}

\subsection{General Information}

Reagents and solvents were purchased from commercial sources and used without further purification unless otherwise specified. Air- and moisture-sensitive liquids and solutions were transferred via syringe or stainless steel cannula. Organic solutions were concentrated by rotary evaporation (BuChi R-3, Surat, India) below $45{ }^{\circ} \mathrm{C}$ at approximately $20 \mathrm{~mm} \mathrm{Hg}$. All non-aqueous reactions were carried out under anhydrous conditions using flame-dried glassware in an argon atmosphere in dry, freshly distilled solvents, unless otherwise noted. Yields refer to chromatographically and spectroscopically $\left({ }^{1} \mathrm{H}-\mathrm{NMR}\right)$ homogeneous materials, unless otherwise stated. Reactions were monitored by thin-layer chromatography (TLC) carried out on $0.15-0.20 \mathrm{~mm}$ silica gel plates (RSGF 254, Yantai, China) using UV light as the visualizing agent. The melting points (m.p.) were measured on a WRS-1B apparatus (Hangzhou, China) and are not corrected. ${ }^{1} \mathrm{H}-\mathrm{NMR}(300 \mathrm{MHz})$ and ${ }^{13} \mathrm{C}-\mathrm{NMR}$ spectra $(75 \mathrm{MHz})$ were obtained with a Bruker AV-300 spectrometer (Karlsruhe, Germany). Chemical shifts are recorded in ppm downfield from tetramethylsilane. J values are given in Hz. Abbreviations used are s (singlet), d (doublet), t (triplet), q (quartet), b (broad) and m (multiplet). 


\subsection{Synthesis}

1-(6-Hydroxy-2,3,4-trimethoxyphenyl)ethanone (4): To a stirring solution of $3(10 \mathrm{~g}, 54.5 \mathrm{mmol})$ in $\mathrm{BF}_{3} \cdot \mathrm{Et}_{2} \mathrm{O}$ $(25 \mathrm{~mL})$ was added $\mathrm{CH}_{3} \mathrm{COOH}(15 \mathrm{~mL})$. After stirring under $\mathrm{N}_{2}$ atmosphere at $85{ }^{\circ} \mathrm{C}$ for $2.5 \mathrm{~h}$, the reaction mixture was allowed to warm to room temperature. Then ice water $(50 \mathrm{~mL})$ was added slowly. The mixture was extracted with ethyl acetate $(30 \mathrm{~mL})$ three times, and the organic layer was dried over $\mathrm{Na}_{2} \mathrm{SO}_{4}$, filtered and concentrated to afford 4 (11.27 g, $92 \%$ yield) as a yellow solid [9]. M.p. $147-148{ }^{\circ} \mathrm{C}$. ${ }^{1} \mathrm{H}-\mathrm{NMR}\left(\mathrm{CDCl}_{3}\right) \delta 6.28(\mathrm{~s}, 1 \mathrm{H}, 5-\mathrm{H}), 4.10(\mathrm{~s}, 3 \mathrm{H}, 2-\mathrm{H}), 3.97(\mathrm{~s}, 3 \mathrm{H}, 3-\mathrm{H}), 3.77(\mathrm{~s}, 3 \mathrm{H}, 4-\mathrm{H}), 2.81(\mathrm{~s}, 3 \mathrm{H}$, 2'-H); ${ }^{13} \mathrm{C}-\mathrm{NMR}\left(\mathrm{CDCl}_{3}\right) \delta 203.5(\mathrm{CO}), 161.9(\mathrm{C}(4)), 160.7(\mathrm{C}(6)), 155.4(\mathrm{C}(2)), 134.5(\mathrm{C}(3)), 108.1(\mathrm{C}(1))$, $96.1(\mathrm{C}(5)), 61.0\left(\mathrm{MeO}-\mathrm{C}\left(2,2^{\prime}\right)\right), 56.4(\mathrm{MeO}-\mathrm{C}(3)), 31.4(\mathrm{MeO}-\mathrm{C}(4))$; ESI-MS: $m / z 227[\mathrm{M}+\mathrm{H}]^{+}$.

1-(6-Hydroxy-2,3,4-trimethoxyphenyl)-3-(4-methoxyphenyl)prop-2-en-1-one (5): 4-Methoxybenzaldehyde $(8 \mathrm{~mL}, 66 \mathrm{mmol}, 1.5$ equiv) was added to a stirring mixture of $4(10 \mathrm{~g}, 44 \mathrm{mmol})$ and $t$-BuOK $(12.32 \mathrm{~g}$, $110 \mathrm{mmol}, 2.5$ equiv) in $\mathrm{EtOH}(150 \mathrm{~mL})$. The reaction mixture was refluxed gently for $4 \mathrm{~h}$ at $85^{\circ} \mathrm{C}$. After being cooled down to room temperature, the reaction mixture was poured into glacial water and the $\mathrm{pH}$ was adjusted to $3 \sim 4$. Then the mixture was filtered and dried to afford $5(13.02 \mathrm{~g}, 86 \%$ yield) as a yellow solid [9]. M.p. ${ }^{135-136}{ }^{\circ} \mathrm{C} .{ }^{1} \mathrm{H}-\mathrm{NMR}\left(\mathrm{CDCl}_{3}\right) \delta 13.78\left(\mathrm{~s}, 1 \mathrm{H}, 6^{\prime}-\mathrm{OH}\right), 7.84(\mathrm{~s}, 2 \mathrm{H}$, 2,3-H), $7.59\left(\mathrm{~d}, J=8.7 \mathrm{~Hz}, 2 \mathrm{H}, 2^{\prime \prime}, 6^{\prime \prime}-\mathrm{H}\right), 6.92\left(\mathrm{~d}, J=8.7 \mathrm{~Hz}, 2 \mathrm{H}, 3^{\prime \prime}, 5^{\prime \prime}-\mathrm{H}\right), 6.29\left(\mathrm{~s}, 1 \mathrm{H}, 5^{\prime}-\mathrm{H}\right), 3.92(\mathrm{~s}, 3 \mathrm{H}$, 2'-H), $3.90\left(\mathrm{~s}, 3 \mathrm{H}, 3^{\prime}-\mathrm{H}\right), 3.86\left(\mathrm{~s}, 3 \mathrm{H}, 4^{\prime}-\mathrm{H}\right), 3.83\left(\mathrm{~s}, 3 \mathrm{H}, 4^{\prime \prime}-\mathrm{H}\right) ;{ }^{13} \mathrm{C}-\mathrm{NMR}\left(\mathrm{CDCl}_{3}\right) \delta 192.3(\mathrm{CO}), 162.1$ (C(4)), $161.0(\mathrm{C}(6)), 160.0\left(\mathrm{C}\left(4^{\prime}\right)\right), 155.4(\mathrm{C}(2)), 143.7(\mathrm{CO}-\mathrm{C}=\mathrm{CH}), 135.5(\mathrm{C}(3)), 130.6\left(\mathrm{C}\left(2^{\prime}, 6^{\prime}\right)\right), 127.9$ $\left(\mathrm{C}\left(1^{\prime}\right)\right), 124.2(\mathrm{CO}-\mathrm{C}=\mathrm{CH}), 114.8\left(\mathrm{C}\left(3^{\prime}, 5^{\prime}\right)\right), 108.3(\mathrm{C}(1)), 96.1(\mathrm{C}(5)), 62.0(\mathrm{MeO}-\mathrm{C}(2)), 61.4(\mathrm{MeO}-\mathrm{C}(3))$, $56.4(\mathrm{MeO}-\mathrm{C}(4)), 55.1\left(\mathrm{MeO}-\mathrm{C}\left(4^{\prime}\right)\right)$; ESI-MS: $m / z 345[\mathrm{M}+\mathrm{H}]^{+}$.

5,6,7-Trimethoxy-2-(4-methoxyphenyl)-4H-chromen-4-one (6): To a solution of 5 (10 g, $29 \mathrm{mmol})$ dissolved in DMSO $(40 \mathrm{~mL})$ was added $\mathrm{I}_{2}(0.74 \mathrm{~g})$ with vigorous stirring at $100{ }^{\circ} \mathrm{C}$. After $2 \mathrm{~h}$, the reaction mixture was poured into crashed ice water $(30 \mathrm{~mL})$. The precipitate was filtered and washed by $10 \%$ aq. $\mathrm{Na}_{2} \mathrm{~S}_{2} \mathrm{O}_{3}$ soln, followed by recrystallization in EtOH to afford $6(8.13 \mathrm{~g}, 82 \%$ yield $)$ as a yellow solid [9]. M.p. $140-141{ }^{\circ} \mathrm{C} .{ }^{1} \mathrm{H}-\mathrm{NMR}\left(\mathrm{CDCl}_{3}\right) \delta 7.82\left(\mathrm{~d}, J=8.9 \mathrm{~Hz}, 2 \mathrm{H}, 2^{\prime}, 6^{\prime}-\mathrm{H}\right), 7.00(\mathrm{~d}, J=8.9 \mathrm{~Hz}, 2 \mathrm{H}$, $\left.3^{\prime}, 5^{\prime}-\mathrm{H}\right), 6.80(\mathrm{~s}, 1 \mathrm{H}, 3-\mathrm{H}), 6.61(\mathrm{~s}, 1 \mathrm{H}, 8-\mathrm{H}), 3.99(\mathrm{~s}, 3 \mathrm{H}, 5-\mathrm{H}), 3.98(\mathrm{~s}, 3 \mathrm{H}, 6-\mathrm{H}), 3.92(\mathrm{~s}, 3 \mathrm{H}, 7-\mathrm{H}), 3.89(\mathrm{~s}$, 3H, $\left.4^{\prime}-\mathrm{H}\right) ;{ }^{13} \mathrm{C}-\mathrm{NMR}\left(\mathrm{CDCl}_{3}\right)$ \& $177.3(\mathrm{C}(4)), 162.4(\mathrm{C}(2)), 161.0\left(\mathrm{C}\left(4^{\prime}\right)\right), 157.7(\mathrm{C}(7)), 154.4(\mathrm{C}(8 \mathrm{a})), 152.3$ $(C(5)), 140.1(C(6)), 127.7\left(C\left(2^{\prime}, 6^{\prime}\right)\right), 123.7\left(C\left(1^{\prime}\right)\right), 114.3\left(C\left(3^{\prime}, 5^{\prime}\right)\right), 112.8(C(4 a)), 106.9(C(3)), 96.3(C(8))$, $62.1(\mathrm{MeO}-\mathrm{C}(5)), 61.6(\mathrm{MeO}-\mathrm{C}(6)), 56.2(\mathrm{MeO}-\mathrm{C}(7))$, $55.4\left(\mathrm{MeO}-\mathrm{C}\left(4^{\prime}\right)\right)$; ESI-MS: $m / z 343[\mathrm{M}+\mathrm{H}]^{+}$.

4',5,6,7-Tetrahydroxyflavone (2): To a solution of 6 (8 g, $23.2 \mathrm{mmol})$ dissolved in $\mathrm{CH}_{3} \mathrm{COOH}(100 \mathrm{~mL})$ was added $40 \% \mathrm{HBr}(50 \mathrm{~mL})$ with stirring at $120^{\circ} \mathrm{C}$ for $24 \mathrm{~h}$. After cooled down to the room temperature, the reaction mixture was poured into ice water $(50 \mathrm{~mL})$. The precipitate was filtered and washed by water and then recrystallized by EtOH to afford 2 ( $5.97 \mathrm{~g}, 90 \%$ yield) as a yellow solid [9]. M.p. 160-141 ${ }^{\circ} \mathrm{C} .{ }^{1} \mathrm{H}-\mathrm{NMR}$ (DMSO- $\left.d_{6}\right) \delta 12.79(\mathrm{~s}, 1 \mathrm{H}, 5-\mathrm{OH}), 10.44(\mathrm{~s}, 1 \mathrm{H}, 7-\mathrm{OH}), 10.30\left(\mathrm{~s}, 1 \mathrm{H}, 4^{\prime}-\mathrm{OH}\right), 8.71$ (s, 1H, 6-OH), 7.90-7.93 (d, 2H, J = 8.8 Hz, 2', 6'-H), 6.90-6.93 (d, 2H, J = 8.8 Hz, 3', 5'-H), $6.78(\mathrm{~s}, 1 \mathrm{H}$, 3-H), 6.73 (s, 1H, 8-H); ${ }^{13} \mathrm{C}-\mathrm{NMR}$ (DMSO-d 6 ) $\delta 182.3(\mathrm{C}(4)), 162.4(\mathrm{C}(2)), 161.0\left(\mathrm{C}\left(4^{\prime}\right)\right), 153.7$ (C(7)), 149.4 $(C(8 a)), 147.3(C(5)), 129.1(C(6)), 128.7\left(C\left(2^{\prime}, 6^{\prime}\right)\right), 121.7\left(C\left(1^{\prime}\right)\right), 116.3\left(C\left(3^{\prime}, 5^{\prime}\right)\right), 104.8(C(4 a)), 102.9(C(3))$, $93.3(\mathrm{C}(8))$; ESI-MS: $m / z 287[\mathrm{M}+\mathrm{H}]^{+}$.

\section{Conclusions}

In summary, we have developed an efficient chemical synthesis of scutellarein (2) in high yield in only four steps. This strategy relies on acetylation, aldolization, cyclization and hydrolysis step, respectively. This synthetic method is effective and it has industrial application value.

Acknowledgments: This work was supported by National Natural Science Foundation of China (No. 81274058, 21302225), China Scholarship Council (No. 201407060046), Natural Science Foundation of Jiangsu Province (BK20151563), the Program for New Century Excellent Talents by the Ministry of Education (NCET-12-0741), 333 High-level Talents Training Project Funded by Jiangsu Province, Six Talents Project Funded by Jiangsu Province 
(2013-YY-010), Technology Innovation Venture Fund by Nanjing University of Chinese Medicine (CX201301), Program for Excellent Talents in School of Pharmacy of Nanjing University of Chinese Medicine (15ZYXET-1), Jiangsu Collaborative Innovation Center of Chinese Medicinal Resources Industrialization (ZDXMHT-1-13), Project Funded by the Priority Academic Program Development of Jiangsu Higher Education Institutions and Project Funded by the Flagship Major Development of Jiangsu Higher Education Institutions (PPZY2015A070).

Author Contributions: Ze-Xi Dong designed the synthetic route, Ze-Xi Dong, Ting Gu, Peng-Xuan Zhang, and Wen-Yu Wu did the experiments, Zhi-Hao Shi wrote this paper, Nian-Guang Li revised this paper.

Conflicts of Interest: The authors declare no conflict of interest.

\section{References}

1. Donnan, G.A.; Fisher, M.; Macleod, M.; Davis, S.M. Stroke. Lancet 2008, 371, 1612-1623. [CrossRef]

2. Cuzzocrea, S.; Riley, D.P.; Caputi, A.P.; Salvemini, D. Antioxidant therapy: A new pharmacological approach in shock, inflammation, and ischemia/reperfusion injury. Pharmacol. Rev. 2001, 53, 135-159.

3. Zhang, J.L.; Che, Q.M.; Li, S.Z.; Zhou, T.H. Study on metabolism of scutellarin in rats by HPLC-MS and HPLC-NMR. J. Asian Nat. Prod. Res. 2003, 5, 249-256. [CrossRef] [PubMed]

4. Che, Q.M.; Chen, Y.; Pan, L.Y.; He, H. Scutellarein's pharmacokinetics in rats. Chin. J. New Drugs 2006, 15, 1557-1561.

5. Qian, L.H.; Shen, M.Z.; Tang, H.; Tang, Y.P.; Zhang, L.; Fu, Y.; Shi, Q.P.; Li, N.G. Synthesis and Protective Effect of Scutellarein on Focal Cerebral Ischemia/Reperfusion in Rats. Molecules 2012, 17, 10667-10674. [CrossRef] [PubMed]

6. Tang, H.; Tang, Y.P.; Li, N.G.; Shi, Q.P.; Guo, J.M.; Shang, E.X.; Duan, J.A. Neuroprotective effects of scutellarin and scutellarein on repeatedly cerebral ischemia-reperfusion in rats. Pharmacol. Biochem. B 2014, 118, 51-59.

7. Qian, L.H.; Li, N.G.; Tang, Y.P.; Zhang, L.; Tang, H.; Wang, Z.J.; Liu, L.; Song, S.L.; Guo, J.M.; Ding, A.W. Synthesis and Bio-Activity Evaluation of Scutellarein as a Potent Agent for the Therapy of Ischemic Cerebrovascular Disease. Int. J. Mol. Sci. 2011, 12, 8208-8216. [CrossRef] [PubMed]

8. Cui, J.M.; Fang, G.; Duan, Y.B.; Liu, Q.; Wu, L.P.; Zhang, G.H.; Wu, S. Total synthesis of scutellarin-7-O-Glucuronide. J. Asian Nat. Prod. Res. 2005, 7, 655-660. [CrossRef] [PubMed]

9. Yen, M.H.; Wu, E.S.C. Chromones and Chromone Derivatives and Uses Thereof. US Patent 0142 211, 29 June 2006.

Sample Availability: Samples of the compounds 2-6 are available from the authors.

(C) 2016 by the authors; licensee MDPI, Basel, Switzerland. This article is an open access article distributed under the terms and conditions of the Creative Commons by Attribution (CC-BY) license (http://creativecommons.org/licenses/by/4.0/). 